\title{
Substrate Specificity in Thiol Dioxygenases
}

Sekotilani Aloi, ${ }^{a}$ Casey G. Davies, ${ }^{a}$ P. Andrew Karplus, ${ }^{b}$ Sigurd M. Wilbanks, ${ }^{c}$ Guy N. L. Jameson ${ }^{\text {ad, }}, *$

${ }^{\text {a }}$ Department of Chemistry, University of Otago, P.O. Box 56, Dunedin 9054. New Zealand.

${ }^{\mathrm{b}}$ Department of Biochemistry and Biophysics, Oregon State University, 2011 Ag \& Life Sciences Building, Corvallis, Oregon 97331, United States.

${ }^{c}$ Department of Biochemistry, University of Otago, P.O. Box 56, Dunedin 9054. New Zealand.

${ }^{d}$ School of Chemistry, Bio21 Molecular Science and Biotechnology Institute, 30 Flemington Road, The University of Melbourne, Parkville VIC 3010, Australia. guy.jameson@unimelb.edu.au

\section{Contents}

3-MPA docking $\quad$ S2

Coupling Efficiency $\quad$ S5

$\begin{array}{ll}\text { Cysteine oxidation by Q62R Pa3MDO } & \text { S7 }\end{array}$

$\begin{array}{ll}\text { Competition Experiments } & \text { S8 }\end{array}$ 


\section{3-MPA docking}

3-MPA was docked into chain c of Pa3MDO from PDB 4TLF as described in the Methods and Materials of the main manuscript. Initial docking of 3-MPA made use of Autodock vina. ${ }^{1}$ 3-MPA was found to interact with Arg168 via the carboxylate but, as expected, the Fe-S bond was not properly modelled and so manual docking was used to produce a better fit (Figure S1). The best fit was found where the sulfur and an $\mathrm{O}$ atom $(\mathrm{O} 2)$ of the 3-MPA coordinated to the iron while both $\mathrm{O}$ atoms formed a salt bridge with Arg168 (Figure S2).

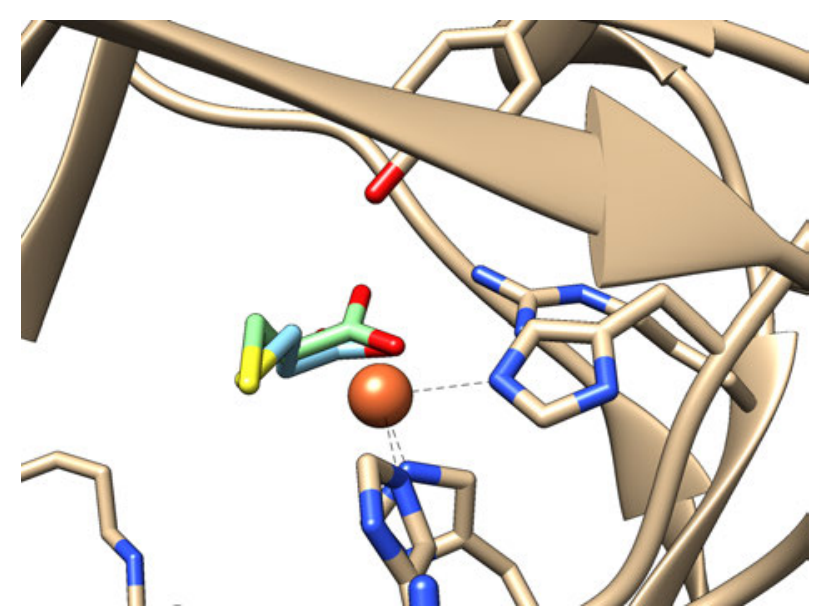

Figure S1. Comparison of 3-MPA docked through Autodock vina (cyan) with the model composed manually (green). 
a)

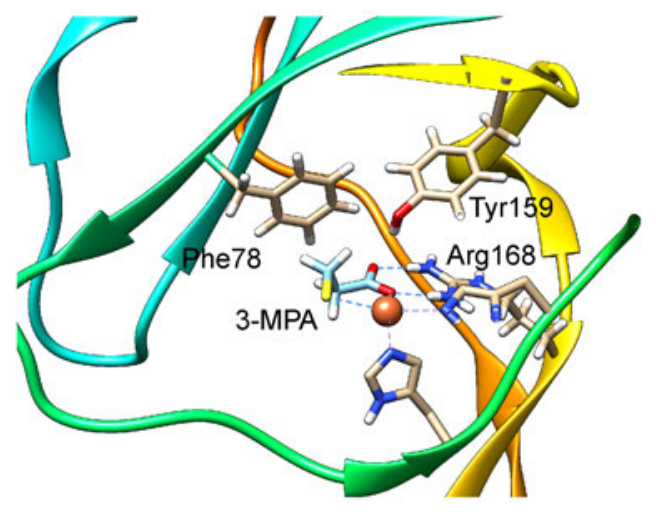

b)

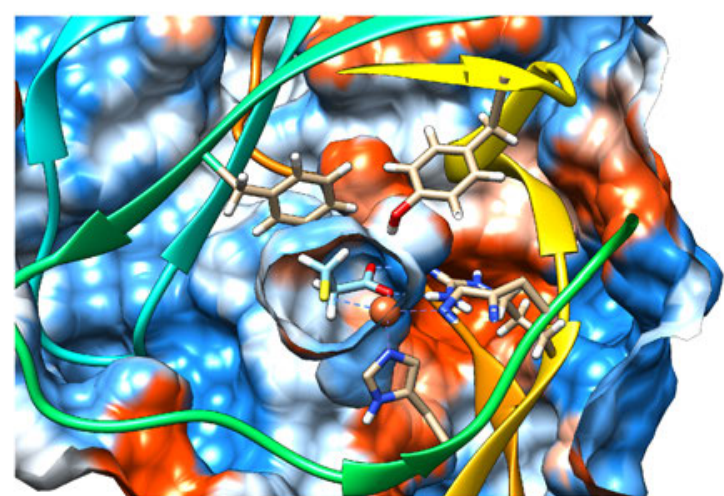

c)

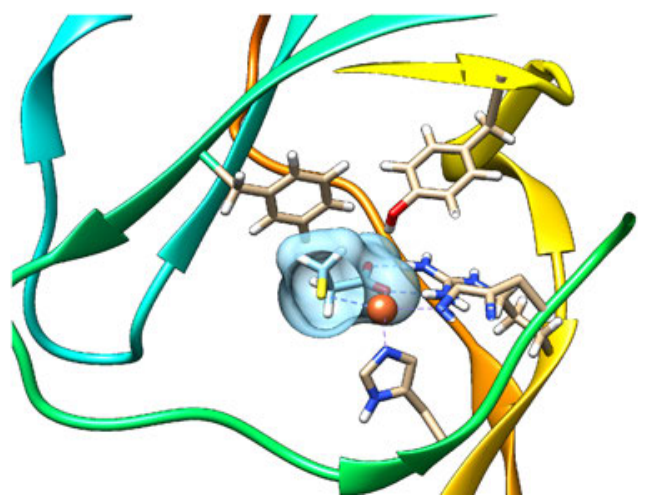

Figure S2. 3-MPA docked into the active site of Pa3MDO. a) Cut away view of the active site showing 3-MPA coordinating to Fe and forming a salt-bridge with Arg168. Phe78 can be seen to make van der Waals contacts with both substrate methylenes. b) The same view showing the protein surface and the active-site pocket the holds 3-MPA. c) The same view showing the surface of 3-MPA is complementary to the pocket shown in b). 
Table S1. Coordinates of the final docked 3-MPA relative to chain c of 4TLF (all water molecules bound to Fe removed).

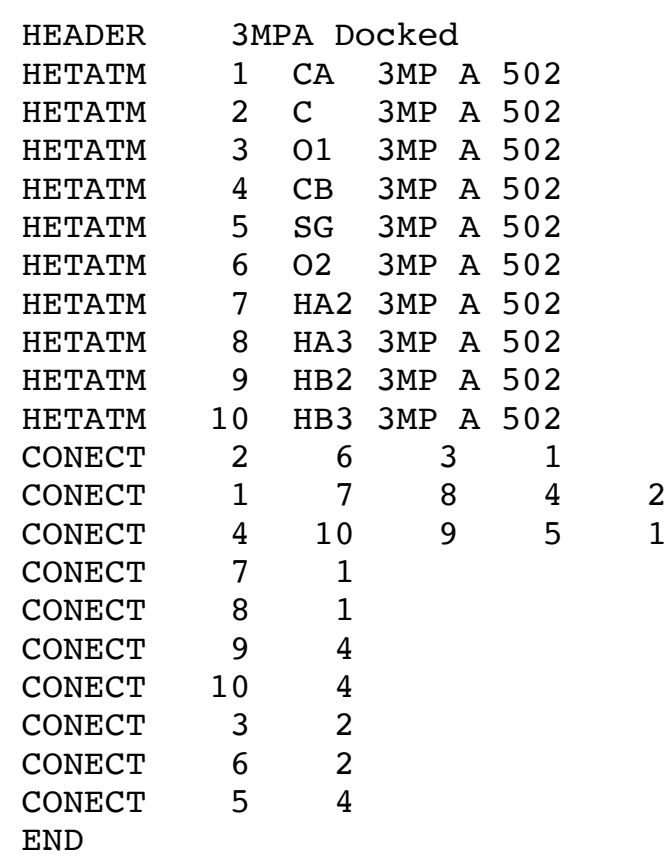

$\begin{array}{rrrrrr}38.272 & 24.653 & 26.474 & 1.00 & 28.81 & \mathrm{C} \\ 37.147 & 25.600 & 26.832 & 1.00 & 36.10 & \mathrm{C} \\ 36.343 & 25.341 & 27.728 & 1.00 & 29.00 & \mathrm{O} \\ 39.612 & 25.262 & 26.903 & 1.00 & 27.16 & \mathrm{C} \\ 40.634 & 25.870 & 25.493 & 1.00 & 28.20 & \mathrm{~S} \\ 37.043 & 26.662 & 26.226 & 1.00 & 33.82 & \mathrm{O} \\ 38.124 & 23.703 & 26.987 & 1.00 & 0.00 & \mathrm{H} \\ 38.278 & 24.487 & 25.397 & 1.00 & 0.00 & \mathrm{H} \\ 39.411 & 26.101 & 27.570 & 1.00 & 0.00 & \mathrm{H} \\ 40.180 & 24.509 & 27.449 & 1.00 & 0.00 & \mathrm{H}\end{array}$




\section{Coupling Efficiency}

The term coupling has become an important concept in the description of the selectivity of reactions and substrate specificity of oxygenases. ${ }^{2-10}$ It provides insight into which active-site residues are critical for interactions between the enzyme and substrate, as well as those important for catalysis. ${ }^{4,8}$ However, despite its extensive use, there is no general consensus on a single interpretation of the term. As a result, there is some inconsistency in the way coupling efficiency is described across the literature. For instance, for the cytochrome P450 enzymes, coupling efficiency is referred to as electron selectivity, ${ }^{4}$ which is the efficiency of electron transfer from NADPH to the P450 enzyme for substrate oxidation. ${ }^{5}$ Definitions include calculating the percent of NADPH used for substrate hydroxylation, taking the ratio of the initial rate of product formation to the initial rate of NADPH oxidation, and calculating moles of product formed per 100 moles of NADPH consumed. , $^{10-11}$ Poor coupling efficiency has been attributed to the loss of electrons from substrate oxidation to the formation of water or reactive oxygen species (ROS) $\mathrm{H}_{2} \mathrm{O}_{2}$ or $\mathrm{O}_{2}{ }^{-10}$ This alternative reaction pathway 'uncouples' electron transfer from substrate oxidation and is referred to as uncoupling. During uncoupled reactions of P450 enzymes, the substrate remains unused at the active-site, while the electrons from NADPH are used for producing either water or ROS. ${ }^{12}$

In contrast to reactions catalyzed by $\mathrm{P} 450$ enzymes, those that are catalyzed by thiol dioxygenases do not involve an external redox co-factor as shown Scheme S1. In the case of thiol dioxygenases, the uncoupled reaction utilizes the substrate but forms a thiol disulfide instead of a sulfinic acid. Because of these differences, the term coupling should be defined slightly differently in terms of thiol dioxygenation. The Pierce group described coupling as the efficiency at which an oxygenase enzyme incorporates 1 mol of $\mathrm{O}_{2}$ into the product. ${ }^{8}$ The group uses the measurements of CSA formation and $\mathrm{O}_{2}$ loss to determine coupling efficiency. In turn, they define $\left(\mathrm{CSA} / \mathrm{O}_{2}\right)$-coupling, under steady-state kinetics, as the ratio of the $k_{\text {cat }}$ determined for CSA formation divided by the $k_{\text {cat }}$ obtained for the rate of $\mathrm{O}_{2}$-consumption as shown in Equation $1 .^{7}$ However, the $\left(\mathrm{CSA} / \mathrm{O}_{2}\right)$-coupling efficiency ${ }^{7-9}$ is also determined using the rate of CSA formation divided by the rate of $\mathrm{O}_{2}$ consumption at a high concentration of other substrate as shown in the following equations, which approximates to $k_{\text {cat: }}$ :

$\left(\frac{C S A}{O_{2}}\right) \cdot$ coupling $=\frac{k_{\text {cat }} \text { of CSA formation }}{k_{\text {cat }} \text { of } O_{2} \text { depletion }} \approx \frac{\text { rate of CSA formation }}{\text { rate of } O_{2} \text { depletion }}$

EqS1

Coupling efficiency $=\frac{\text { rate of sulfinate formation }}{\text { rate of thiol depletion }}=\frac{\text { amount of sulfinate formed }}{\text { amount of thiol depleted }}$

EqS2 
a)

$$
\mathrm{O}_{2} 2 \mathrm{e}^{-}+2 \mathrm{H}^{+}
$$

P450

$$
\longrightarrow 2 \mathrm{H}_{2} \mathrm{O} \text { or } \mathrm{H}_{2} \mathrm{O}_{2} \text { or } \mathrm{HO}_{2} \text { coupled }
$$

b)

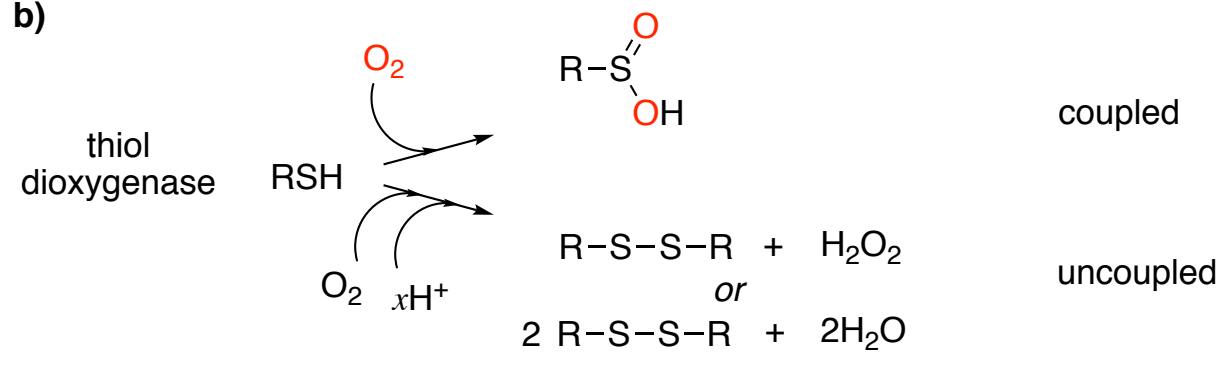

Scheme S1. A comparison of the coupled and uncoupled reactions of a) P450 and b) thiol dioxygenases.

Although measurement of $\mathrm{O}_{2}$ is a valid way for determining rates, ${ }^{8}$ various issues can be encountered with this approach when not carefully designed. These measurements are not always accurate as $\mathrm{O}_{2}$ consumption at a Clark-type electrode that is typically used, depends on many factors including $\mathrm{pH}$, temperature, stirring, electrode response time, and even the reaction volume. Additionally, if substrate depletion and product formation are measured separately, it is crucial that the reaction conditions are exactly the same. In the present study, these potential issues are circumvented by measuring the loss of thiol substrate instead of $\mathrm{O}_{2}$ and using ${ }^{1} \mathrm{H}$ NMR analysis, which has the benefits of following the changes in substrate and product simultaneously under identical conditions. This, together with the already described Ellman's assay for following thiol substrate depletion provides a robust method for the accurate determination of reaction rates. Therefore, the coupling efficiency is described here as the efficiency at which the thiol substrate couples to product-formation, and is determined using EqS2. The use of EqS2 is justified because only two products are formed during the reaction, and rate data suggest that substrate depletion and product formation occur in parallel, meaning that the ratio of product to substrate is the same over time. 


\section{Cysteine oxidation by Q62R Pa3MDO}

Cysteine is not the main substrate and thus oxidation by Q62R Pa3MDO was studied over a few pHs to compare with wild type. ${ }^{13}$
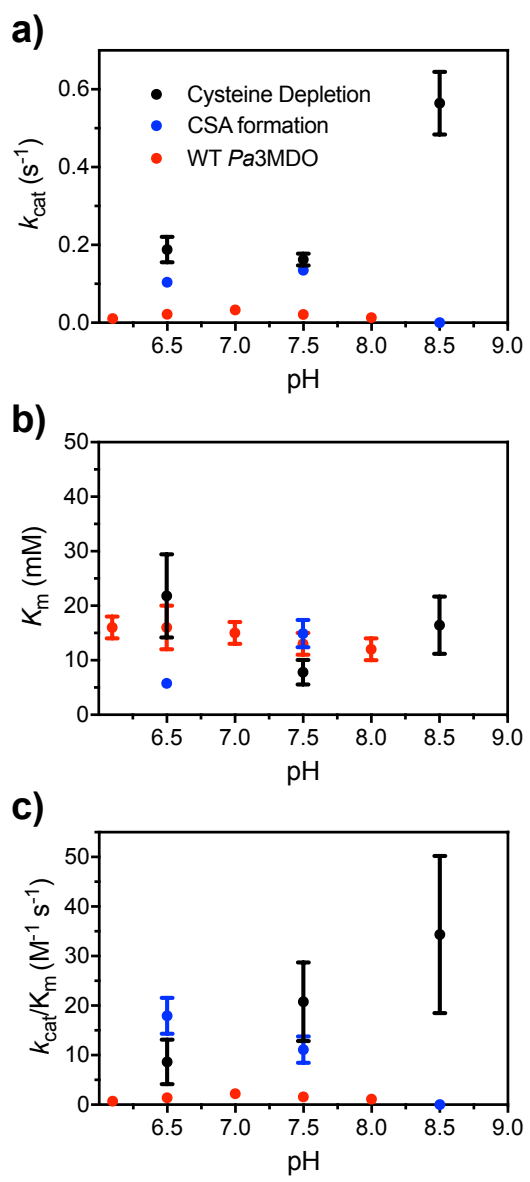

Figure S3. pH dependent kinetic parameters for Q62R Pa3MDO sulfination of cysteine. Values of $k_{\text {cat }}, K_{\mathrm{m}}$, and $k_{\mathrm{cat}} / K_{\mathrm{m}}$ for cysteine depletion via Ellman's assay (black) and CSA formation via HPLC-ELSD (blue). The kinetic data for WT Pa3MDO is also presented ${ }^{13}$ (red). 


\section{Competition Experiments}

Reactions of Q62R Pa3MDO (30 $\mu \mathrm{M})$ with equimolar mixtures of substrates $(10 \mathrm{mM})$ were left for 10 hours and studied by ${ }^{1} \mathrm{H}$ NMR to determine which substrate was preferred. As the kinetic parameters also indicate (Table 2, main manuscript), the order of reactivity is 3MPA $>$ (R)-MSA $>$ L-cys.
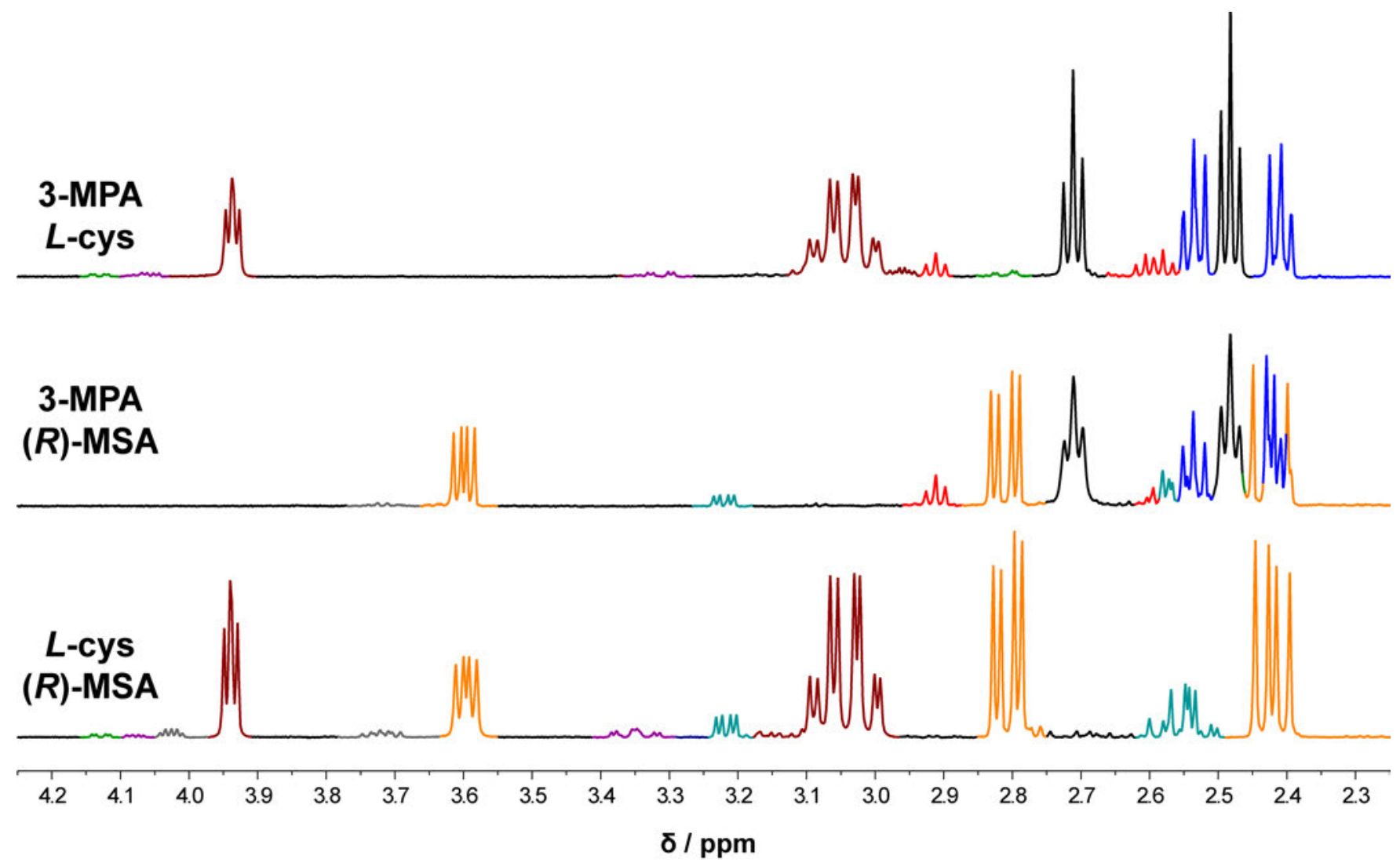

Figure S4. ${ }^{1} \mathrm{H}$ NMR spectra of reactions of Q62R Pa3MDO with 3-MPA and L-cys (top), 3-MPA and (R)-MSA (middle) and L-cys and (R)-MSA (bottom). 3-MPA (black), 3-SPA (blue), 3-MPA disulfide (red), L-cys (maroon), CSA (green), cystine (purple), (R)-MSA (orange), (R)-SSA (turquoise). 


\section{References}

1. Trott, O.; Olson, A. J., AutoDock Vina: Improving the speed and accuracy of docking with a new scoring function, efficient optimization, and multithreading. J. Comput. Chem. 2010, 31 (2), 455461.

2. White, R. E.; McCarthy, M. B.; Egeberg, K. D.; Sligar, S. G., Regioselectivity in the cytochromes P-450: control by protein constraints and by chemical reactivities. Arch. Biochem. Biophys. 1984, $228(2), 493-502$.

3. Jouanneau, Y.; Meyer, C.; Jakoncic, J.; Stojanoff, V.; Gaillard, J., Characterization of a naphthalene dioxygenase endowed with an exceptionally broad substrate specificity toward polycyclic aromatic hydrocarbons. Biochemistry 2006, 45 (40), 12380-91.

4. Traylor, M. J.; Chai, J.; Clark, D. S., Simultaneous measurement of CYP1A2 activity, regioselectivity, and coupling: Implications for environmental sensitivity of enzyme-substrate binding. Arch. Biochem. Biophys. 2011, 505 (2), 186-193.

5. O'Keefe, D. P.; Lau, S. M.; Harder, P. A., Efficiency and substrate specificity of Streptomyces griseolus cytochromes P-450SUI and P-450SU2 mono-oxygenase reactions. Biochem. Soc. Trans. 1993, 21 (4), 1073-7.

6. Cirino, P. C.; Arnold, F. H., Regioselectivity and Activity of Cytochrome P450 BM-3 and Mutant F87A in Reactions Driven by Hydrogen Peroxide. Adv Synth Catal. 2002, 344 (9), 932-937.

7. Li, W.; Pierce, B. S., Steady-state substrate specificity and O(2)-coupling efficiency of mouse cysteine dioxygenase. Archives of biochemistry and biophysics 2015, 565, 49-56.

8. Li, W.; Blaesi, E. J.; Pecore, M. D.; Crowell, J. K.; Pierce, B. S., Second-sphere interactions between the C93-Y157 cross-link and the substrate-bound Fe site influence the $\mathrm{O}(2)$ coupling efficiency in mouse cysteine dioxygenase. Biochemistry 2013, 52 (51), 9104-19.

9. Crowell, J. K.; Li, W.; Pierce, B. S., Oxidative Uncoupling in Cysteine Dioxygenase Is Gated by a Proton-Sensitive Intermediate. Biochemistry 2014, 53 (48), 7541-7548.

10. Lim, J. B.; Barker, K. A.; Eller, K. A.; Jiang, L.; Molina, V.; Saifee, J. F.; Sikes, H. D., Insights into electron leakage in the reaction cycle of cytochrome P450 BM3 revealed by kinetic modeling and mutagenesis. Protein Sci. 2015, 24 (11), 1874-1883.

11. Kolev, J. N.; Zaengle, J. M.; Ravikumar, R.; Fasan, R., Enhancing the Efficiency and Regioselectivity of P450 Oxidation Catalysts via Unnatural Amino Acid Mutagenesis. Chembiochem. 2014, 15 (7), 1001-1010.

12. Kadkhodayan, S.; Coulter, E. D.; Maryniak, D. M.; Bryson, T. A.; Dawson, J. H., Uncoupling oxygen transfer and electron transfer in the oxygenation of camphor analogues by cytochrome P450-CAM. Direct observation of an intermolecular isotope effect for substrate C-H activation. $J$. Biol. Chem. 1995, 270 (47), 28042-8.

13. Fellner, M.; Aloi, S.; Tchesnokov, E. P.; Wilbanks, S. M.; Jameson, G. N. L., Substrate and pHDependent Kinetic Profile of 3-Mercaptopropionate Dioxygenase from Pseudomonas aeruginosa. Biochemistry 2016, 55 (9), 1362-1371. 